\title{
Erratum to: Effect of calorie restriction and refeeding on skin wound healing in rats
}

\author{
Nicole D. Hunt • Garrick D. Li • Min Zhu • \\ Marshall Miller • Andrew Levette • \\ Mark E. Chachich • Edward L. Spangler • \\ Joanne S. Allard • Dong-Hoon Hyun • \\ Donald K. Ingram • Rafael de Cabo
}

Published online: 29 December 2011

(C) American Aging Association 2011

\section{Erratum to: Age \\ DOI 10.1007/s11357-011-9321-6}

Authorship on this paper should also include Marshall Miller, Laboratory of Experimental Gerontology, NIA, $\mathrm{NIH}$, Baltimore, MD whose contributions to statistical analysis and interpretation helped formulate this publication.

Corrected list of authors is shown above and below.

The online version of the original article can be found at http://dx. doi.org/10.1007/s11357-011-9321-6.

N. D. Hunt · G. D. Li • M. Zhu • M. Miller · A. Levette

E. L. Spangler $\cdot$ J. S. Allard $\cdot$ R. de Cabo $(\bowtie)$

Laboratory of Experimental Gerontology,

National Institute on Aging, National Institutes of Health,

Baltimore, MD 21224, USA

e-mail: decabora@mail.nih.gov

M. E. Chachich

Department of Psychology, Towson University,

Towson, MD 21252, USA

D.-H. Hyun

Department of Life Science, Ewha Womans University,

11-1 Daehyeon-dong Seodaemun-gu,

Seoul 120-750, South Korea

D. K. Ingram

Nutritional Neuroscience and Aging Laboratory,

Pennington Biomedical Research Center,

Louisiana State University System,

Baton Rouge, LA 70808-412, USA 